\title{
Nesting and Berth Allocation by an Expert System Using Heuristic Search
}

\author{
Dongmok Sheen ${ }^{1}$, Yoonho Seo ${ }^{*}$ \\ ${ }^{1}$ School of Naval Architecture and Ocean Engineering, University of Ulsan, Ulsan, Korea \\ ${ }^{2}$ School of Industrial Management Engineering, Korea University, Seoul, Korea \\ Email: dmsheen@ulsan.ac.kr, *yoonhoseo@korea.ac.kr
}

How to cite this paper: Sheen, D. and Seo, Y. (2017) Nesting and Berth Allocation by an Expert System Using Heuristic Search. Journal of Software Engineering and Applications, 10, 311-323. https://doi.org/10.4236/jsea.2017.104018

Received: February 14, 2017

Accepted: April 3, 2017

Published: April 6, 2017

Copyright () 2017 by authors and Scientific Research Publishing Inc. This work is licensed under the Creative Commons Attribution International License (CC BY 4.0).

http://creativecommons.org/licenses/by/4.0/

\section{cC) (i) Open Access}

\begin{abstract}
Nesting is a common problem in industries such as shipbuilding, auto-maker, clothing, shoe-making, and furniture, in which various parts are cut off from a stock or stocks while minimizing the wastes or maximizing the utilization of the stock. Berth allocation at seaside is also considered one form of two dimensional nesting problems, in which a ship is assigned a location for service during a certain time slot. This paper presents an expert system using a heuristic search method for nesting problems. The parts and stocks are represented by pixels with which utility function is used to evaluate current state in search tree. The system is developed in CLIPS, an expert system shell and applied to various example problems with different constraints and to a berth allocation example to illustrate its applicability under different conditions.
\end{abstract}

\section{Keywords}

Nesting, Berth Allocation, Heuristic Search, CLIPS

\section{Introduction}

Nesting can be defined as a problem of packing various shapes as close as possible in a limited space in order to minimize the wastes. Cutting parts from raw planar materials in shipbuilding, automobiles, furniture, shoes, clothing, and paper companies, or storing parts in space are some example nesting problems. Since nesting is an NP-complete problem [1], heuristics are used in solving the problem. Shapes are usually sorted first by size and then approximated as simple convex polygons with which algorithms such as NFP (Nofit Polygon) [2] are used, or represented as pixels with which algorithms such as BLF (bottom-left filling) algorithm [3] [4] are used. In special application such as laser cutting, minimization of cycle time by optimal cutting sequence as well waste is also considered [5]. 
Spatial scheduling is another well-known nesting problem in which one axis is time. Three-dimensional spatial scheduling, where a part is represented as a prism with service time as height, minimizing make span at block assembly shop is reported [6]. Reference [7] used genetic algorithm to solve a two-dimensional spatial scheduling problem for blocks with growing lengths as assembly progresses at pre-erection area in shipbuilding. Berth allocation problem is a two-dimensional spatial scheduling problem for seaside operations planning, in which a berth is allocated for each ship to load and unload freight within a specified service time [8].

This paper presents an expert system utilizing heuristic search to solve 2-dimensional nesting problems with different constraints and a berth allocation problem. Expert system approach is used for nesting and berth allocation problems since they have discrete number of possible solutions and the performance can be improved at implementation stage. Compared with algorithmic approaches, the performance of the system can be improved by easily adding new rules at implementation stage. For berth allocation problem, for instance, we can add more rules depending on the situation of the quay and scheduling rules.

\section{Backgrounds}

In order to solve nesting problems, shapes to be nested are usually represented by pixels or approximate polygons. It is easy to represent shapes in pixels and the overlapping between shapes can be easily checked, but they require lots of memory and computing time to represent the shapes accurately. On the other hand, the methods based on approximate polygons need less memory to store data for edges and vertices of polygons, but algorithm for checking overlaps becomes complicated.

When pixels are used, after defining the resolution, a shape is represented by an enclosing rectangle(ER). Usually the boundary and inside the shape are represented by " 1 " values while the other part of ER is represented by " 0 " values as in Figure 1(a) [4]. Some researchers assign different values for the pixels on boundary than the ones inside (Figure 1(b)) so that the types of overlapping (boundary-boundary, boundary-inside, or inside-inside) between two shapes can be easily identified [1] [3]. In pixel-based approaches, BLF algorithm [3] [4] is frequently used. It scans vertically from the left bottom pixel to the top pixel and then repeats from the right adjacent pixel at the bottom to the top until they find an empty space for the shape to be assigned. Among the approaches based on approximate polygons, NFP algorithms [2] are well known. Figure 2 illustrates an NFP method. When polygon B moves along the edges of polygon A, the trajectory of a particular point $\mathrm{P}$ of $\mathrm{B}$ becomes NFP, NAB. When we lay shape $B$ at a position, if $P$ of $B$ enters inside the $N A B$, it means the shape $B$ overlaps the shape A. When there are concave shapes or shapes with holes, NFP algorithm becomes complicated.

NFP is an efficient method to check collision between two parts, but it is not used to check overlaps among multiple shapes more than two. 


\begin{tabular}{|l|l|l|l|l|l|l|l|l|}
\hline 0 & 0 & 0 & 0 & 0 & 0 & 0 & 0 & 0 \\
\hline 0 & 1 & 1 & 1 & 1 & 1 & 0 & 0 & 0 \\
\hline 0 & 1 & 1 & 1 & 1 & 1 & 1 & 0 & 0 \\
\hline 0 & 1 & 1 & 1 & 1 & 1 & 1 & 1 & 0 \\
\hline 0 & 1 & 1 & 1 & 1 & 1 & 1 & & 0 \\
\hline 0 & 1 & 1 & 1 & 1 & 1 & 1 & & 0 \\
\hline 0 & 1 & 1 & 1 & 1 & 1 & 1 & & 0 \\
\hline 0 & 0 & & & 0 & 1 & 1 & & 0 \\
\hline 0 & 0 & 0 & 0 & 0 & 0 & 0 & 0 & 0 \\
\hline
\end{tabular}

(a)

\begin{tabular}{|l|l|l|l|l|l|l|l|l|}
\hline 0 & 0 & 0 & 0 & 0 & 0 & 0 & 0 & 0 \\
\hline 0 & 1 & 1 & 1 & 1 & 1 & 0 & 0 & 0 \\
\hline 0 & 1 & 3 & 3 & 3 & 1 & 1 & 0 & 0 \\
\hline 0 & 1 & 3 & 3 & 3 & 3 & 1 & 1 & 0 \\
\hline 0 & 1 & 3 & 3 & 3 & 3 & 3 & 1 & 0 \\
\hline 0 & 1 & 3 & 3 & 3 & 3 & 3 & 1 & 0 \\
\hline 0 & 1 & 1 & 1 & 1 & 1 & 3 & 1 & 0 \\
\hline 0 & 0 & 1 & 1 & 0 & 1 & 1 & & 0 \\
\hline 0 & 0 & 0 & 0 & 0 & 0 & 0 & 0 & 0 \\
\hline
\end{tabular}

(b)

Figure 1. Pixel representations. (a) Binary pixels; (b) Pixels having different values inside.

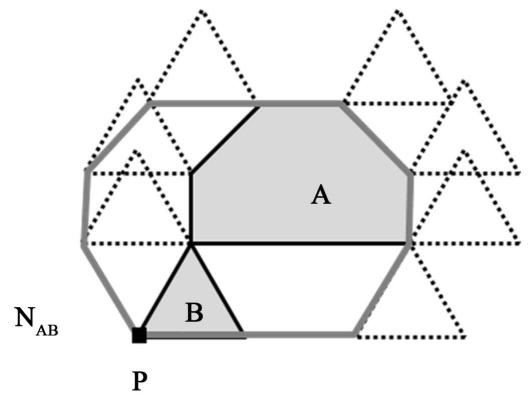

Figure 2. Nofit polygon.

Nesting on blank with irregular boundaries is another research topic [2] [9] [10]. In shipbuilding industry, the parts with the same height are grouped together and cut from a flat bar to manufacture blocks [11]. This condition makes the problem easier since the number of possible positions is greatly reduced and only 4 configurations of a part $\left(0^{\circ}, 180^{\circ}\right.$, and their mirroring shapes) need to be considered. In Lee and Park's research, the parts are sorted first by size in descending order and then each part is added to the layout in that order. Since similar parts are abundant in shipbuilding, Lee et al. [12] paired parts in an effort to simplify the nesting algorithm.

Berth allocation problem is to assign the vessels needing services at specific time-windows at specific locations at quay. It is different from general nesting problem in that each shape is rectangle ("service time $\mathrm{x}$ length") with Earliest start time (ES) and Latest finish time (LF), between which the shape must be located. Problems can be categorized depending on the nature of arrival time, service time, type of quays, etc. Usually vessel's length, draft, expected time of arrival, and service time are given. The layout of quay can be discrete in which vessels can only be assigned at discrete positions, continuous in which vessels can be assigned any positions, or hybrid [8]. A quay may have several water depths to accommodate ships with different drafts. Multiple quays are also considered in allocating berths [13].

Both pixel-based methods and approximate polygon methods have advantages and disadvantages. Pixel-based approaches are easier to implement and can nest 
concave shapes, but they require lots of memory for accuracy. Approximate polygon methods are efficient for convex shapes but it becomes complicated for concave shapes. Expert systems can be a useful nesting tool in that it can easily adopt different types of constraints depending on the application domains and its performance can be easily improved without re-coding as more rules are added.

\section{Nesting Expert System}

\subsection{Data Structure}

In this research nesting is done by an expert system using heuristic search. The shapes are represented by pixels. Figure 3 shows a search tree used in this research. Each node represents a state of layout and as level deepens by one the number of shapes laid increases by one. The root node represents the blank state. At the goal node the number of shapes equals to the number of shapes to be laid. At each node a child node having the maximum value of utility function, which evaluates a node's likelihood of success, is open. One node can have multiple children nodes depending on the number of shapes and their positions, and orientations including mirroring images. In Figure $3, b_{\mathrm{ijk}}$ denotes the i-th part, at the $\mathrm{j}$-th position with the $\mathrm{k}$-th orientation. Even though each part can have infinite number of orientations plus their mirroring images provided the problem domain does not differentiate the front from the backside, the number of orientations is limited to reduce the solution space. The nodes with duplicate orientations due to symmetry are not created.

$\mathrm{N}_{\mathrm{r}}(\mathrm{x})$ number of children nodes are created at node $\mathrm{x}$ for the $\mathrm{r}$-th shape to be laid on the board $B\left(b_{1^{* *}}, b_{2^{*}}, \ldots, b_{r-1^{*}}\right)$ on which ( $\left.r-1\right)$ shapes are already laid. Equation (1) shows the number of children at node $\mathrm{x}$ where $\mathrm{np}$, no are the number of possible positions and orientations for br, and $B\left(b_{1^{* *}}, b_{2^{* *}}, \ldots, b_{r-1^{* *}}\right)$ is denoted as $\operatorname{Br}-1(\mathrm{x})$.

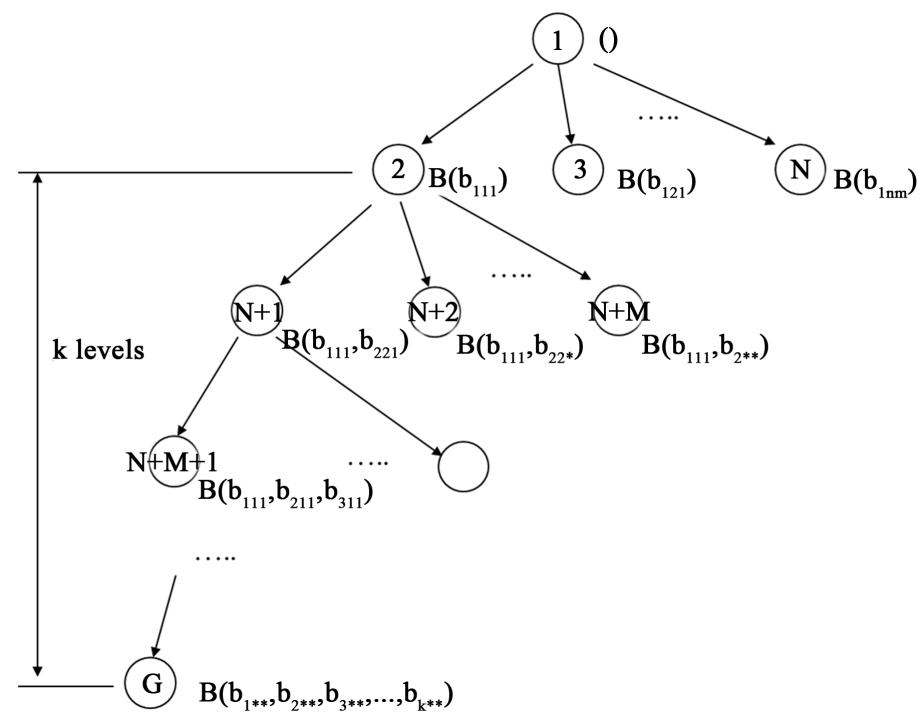

Figure 3. Search tree. 
$N_{r}(x)=\sum_{i=1}^{n_{p}} \sum_{j=1}^{n_{o}} \operatorname{chk}\left(b_{r i j}, B_{r-1}(x)\right)$ where $\operatorname{chk}(b, B)=\left\{\begin{array}{l}0, \text { if } b \text { overlaps } B \\ 1, \quad \text { otherwise }\end{array}\right.$

The following shows the templates of a shape and a node written in CLIPS. A shape has sister patterns created by its orientations, which are stored in the slot orientation. A node keeps a list of shapes already assigned in tile-list with their orientations, positions (row, col) in tile-orient-list, tile-position-row, and tileposition-col which correspond to the subscript of $b_{i j k}$ in Figure 3. Therefore, tilelist, tile-orient-list, tile-position-row, and tile-position-col are empty at rootnode.

(deftemplate MAIN::tile

(slot tile-id (type NUMBER))

(slot cardinality (type NUMBER))

(multislot orientation (type FACT-ADDRESS)))

(deftemplate MAIN::node

(slot node-id (type NUMBER)); natural number

(slot parent (type NUMBER)); If it is 0, no parent

(multislot tile-list (type FACT-ADDRESS))

(multislot tile-orient-list (type FACT-ADDRESS))

(multislot tile-position-row (type NUMBER))

(multislot tile-position-col (type NUMBER)))

The representation of a shape differs depending on the applications. The shapes are represented in pixels for general nesting problems. In berth allocation problems, however, only dimensional properties are stored since the shapes are rectangles with length of ship as width and service time as height. The orientations of shapes are not considered. Instead, the properties such as ES, LF, service time, and length are stored. The following shows the templates of a shape and a node for berth allocation problem written in CLIPS. A shape does not have sister patterns since orientation is not considered, but has the length of the vessel in length, service time in duration, ES, and LF. Therefore, a node does not have tile-orient-list slot as in general nesting problem for orientations of shapes already assigned.

(deftemplate MAIN::tile

(slot tile-id (type NUMBER))

(slot length (type NUMBER))

(slot duration (type NUMBER))

(slot ES (type NUMBER))

(slot LF (type NUMBER)))

(deftemplate MAIN::node

(slot node-id (type NUMBER)); natural number

(slot parent (type NUMBER)); If it is 0 , no parent

(multislot tile-list (type FACT-ADDRESS))

(multislot tile-position-row (type NUMBER))

(multislot tile-position-col (type NUMBER)))

In addition to the information about nodes, the global database also comprises 
open-list keeping the ordered list of nodes to open.

\subsection{Rules}

Rule base consists of rules and the functions related. Rule: initialize sorts shapes by size in descending order. It also creates sister patterns of shapes generated from different orientations and mirroring. The following shows a pseudo-code of Rule: initialize.

Rule: initialize

IF (initial-condition)

THEN

Read shapes and call it $\{s\}$

Sort shapes by size in descending order, and call it $\left\{\mathrm{s}^{*}\right\}$

Create a root node, and call it $\mathrm{n}_{0}$.

Let open-list $=\left\{\mathrm{n}_{0}\right\}$

Rule: open-node is the basic rule to expand the search tree. It gets the first node in open-list and opens it. The children nodes are created and added to open-list as it is open. When adding the children nodes to open-list, they are sorted by the value of utility function and added to the front of open-list to enable depth-first search. The following shows a pseudo code of Rule: open-node.

\section{Rule: open-node}

IF open-list $==\left\{\mathrm{n}_{\mathrm{i}}, \mathrm{REST}\right\}$

THEN

Expand the node ni and save the result as $\{\mathrm{N}\}$.

Sort $\mathrm{N}$ in descending order of utility function value and save it as $\left\{\mathrm{N}^{\star}\right\}$.

Update the set open-list by prepending $\mathrm{N}^{\star}$ to REST.

Let open-list $=\left\{\mathrm{N}^{*}, \mathrm{REST}\right\}$

The following shows the condition part of Rule: open-node rule written in CLIPS. CLIPS use pattern matching in finding the applicable rules at current state.

(defrule MAIN::open-node

?op <- (open-list (nodes ?nid \$?rest-n))

?cn<- (node (node-id ?nid) (parent ?p) (tile-list \$?tlist)

(tile-orient-list \$?torlist) (tile-position-row \$?prlist) (tile-position-col \$?pclist))

(lay-tile-list \$?tlist ?t \$?rest-t)

$=>$....)

This rule expands the first node(?nid) from open-list. The children nodes correspond to all possible positions and orientations (including mirroring images) of the next shape to be assigned. In general, as the problem space grows, the search space grows exponentially. In order to expedite searching, the children nodes are sorted by a utility function in descending order. In general nesting, the utility function measures the sum of overlapping areas between the MER (minimum enclosing rectangle) of the shape and the MER of each shape already laid. Equation (2) shows the utility function for general 2D nesting problem, 
where the function count () counts the number of pixels. $B_{r-1}(x)$ is current layout at node $x, b_{I^{*}}$ indicates the rth shape at certain position and orientation.

$$
u\left(B_{r-1}(x), b_{r^{* *}}\right)=\sum_{b_{k^{* *} \in B_{r-1}}(x)} \operatorname{count}\left(\operatorname{MER}\left(b_{k^{* *}}\right) \bigcap \operatorname{MER}\left(b_{r^{* *}}\right)\right)
$$

The bigger value of utility function means that there are more possibilities for shapes to be closely packed and therefore to get better solution. In calculating the utility function, the four corners of the shape can be easily calculated by offsetting the position. The following pseudo code shows the count function, where $\left(\mathrm{x}_{\mathrm{i}}, \mathrm{y}_{\mathrm{i}}\right)$ and $\left(\mathrm{x}_{\mathrm{ip}}, \mathrm{y}_{\mathrm{ip}}\right)$ are the locations of the upper-left corner the lower-right corners of MER of shape i. In Figure 4, count function counts the number of overlapping pixels, shown in grey color, between two MERs.

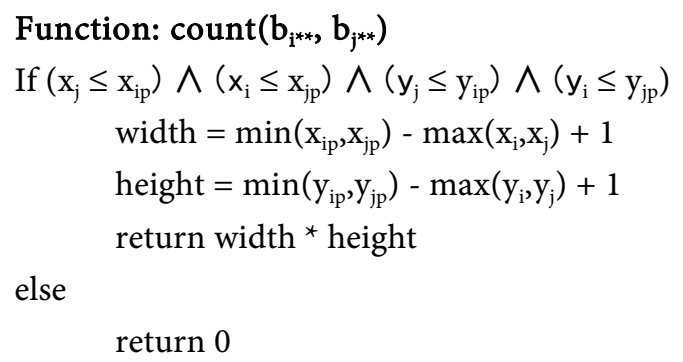

In berth allocation problems, all shapes are rectangles with service time and the length of vessel as height and width respectively. Figure 5 shows a shape in berth allocation problem. Therefore, overlapping between MERs is not allowed. Instead, nodes are sorted by ES in ascending order, by length in descending order, and by LF in ascending order to minimize make span and to maximize the utilization of quay area.

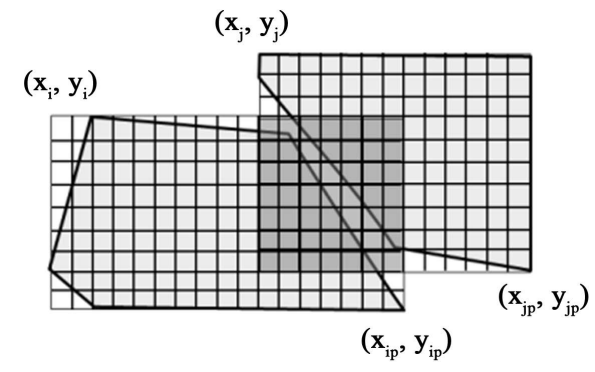

Figure 4. Graphical representation of the evaluation function.

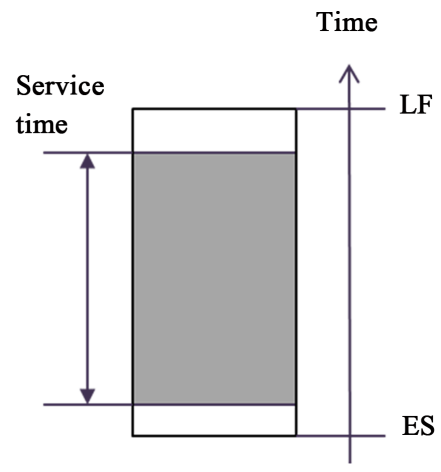

Figure 5. A shape in berth allocation problem. 
Rule: goal checks if the goal state is reached. The following shows the condition part of the rule in CLIPS. The goal node is reached if the number of shapes (\$?tlist) to be laid equals to the number of shapes already laid.

(defrule MAIN::goal

?open <- (open-list (nodes ?nid \$?list))

(lay-tile-list \$?tlist)

(node (node-id ?nid) (tile-list \$?tlist) (tile-orient-list \$?torlist)

(tile-position-row \$?prlist) (tile-position-col \$?pclist))

$=>\ldots)$

\section{Example Problems}

\subsection{Nesting Allowing Mirroring and Rotations}

This example is a general nesting problem in which eight sister patterns (four orientations and their mirroring patterns) for each shape are considered. At initial stage, maximum of eight sister patterns are created for each shape without duplicates. At every node, children nodes are generated for a part for possible positions with all sister patterns are considered to lay the part. The children nodes are sorted in descending order of the values of the utility function shown in Equation (2). Figure 6 shows the result of nesting on $35 \times 20$ size blank.

It is difficult for approximate polygon methods, which are based on convex polygons, to nest a part inside another hollow part. Figure 6 shows that this method does not have that kind of limitations. The solution in Figure 6 was found at the 133,574 th node.

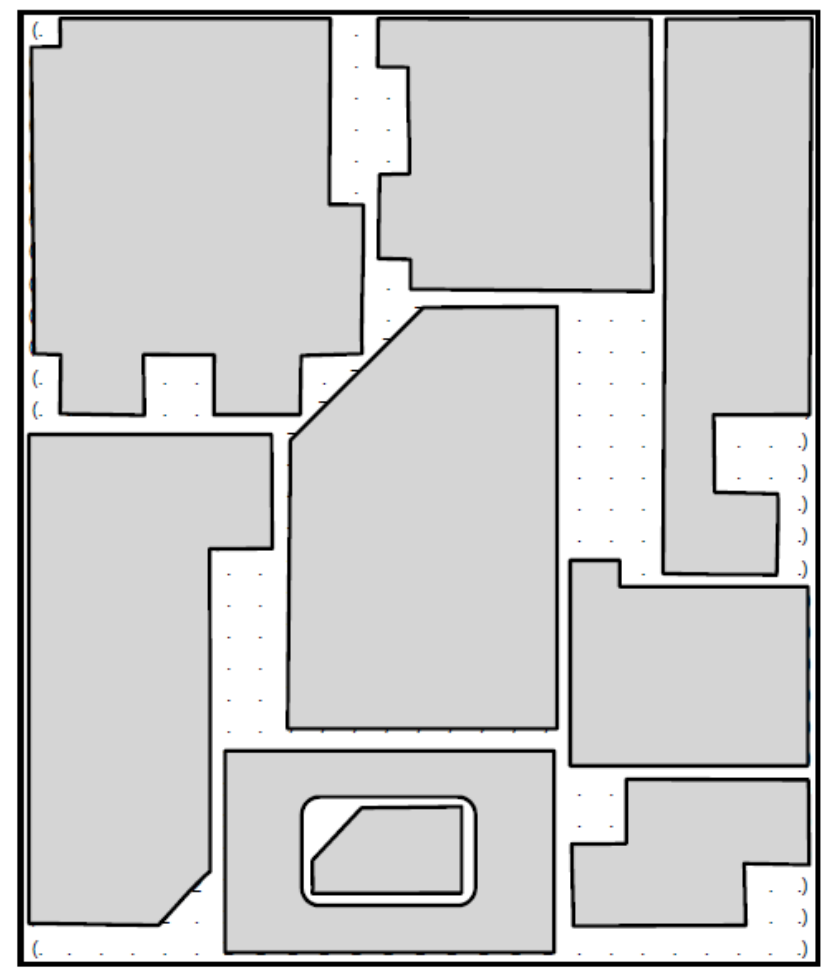

Figure 6. A solution for Example 4.1. 


\subsection{Cutting Parts with Equal Height from a Strip in Shipbuilding}

In shipbuilding shapes are grouped by height and the parts with the same height are cut from a flat bar to build blocks. There are four sister patterns $\left(0^{\circ}, 180^{\circ}\right.$ and their mirroring images) for a shape. In this case, only the order of shapes and their orientations are important, since we would put neighboring shapes as close as possible. For $\mathrm{N}$ parts, there are $\mathrm{N} ! \mathrm{x} 4 \mathrm{~N}$ alternative layouts. In this research, the shapes are sorted by size first for searching order but a shape can be laid at any place. Only the overlapping of the adjacent boundaries of two shapes is considered by utility function. Figure 7 shows example shapes to be cut from the flat bar and Figure 8 shows the result. Since only the left and the right boundaries of shapes are important in this application, the shapes are further simplified to enhance the performance. In Figure $7(\mathrm{~b})$, the number in a pixel greater than "1" represents the width. There are $8 ! \times 48$ (about 2.6 billions) alternative layouts and the solution was found at the $1,258,181$ th node.

\subsection{Nesting on Blank with Irregular Boundaries}

Nesting on irregular stock is another research topic [2] [9] [10]. In our system,
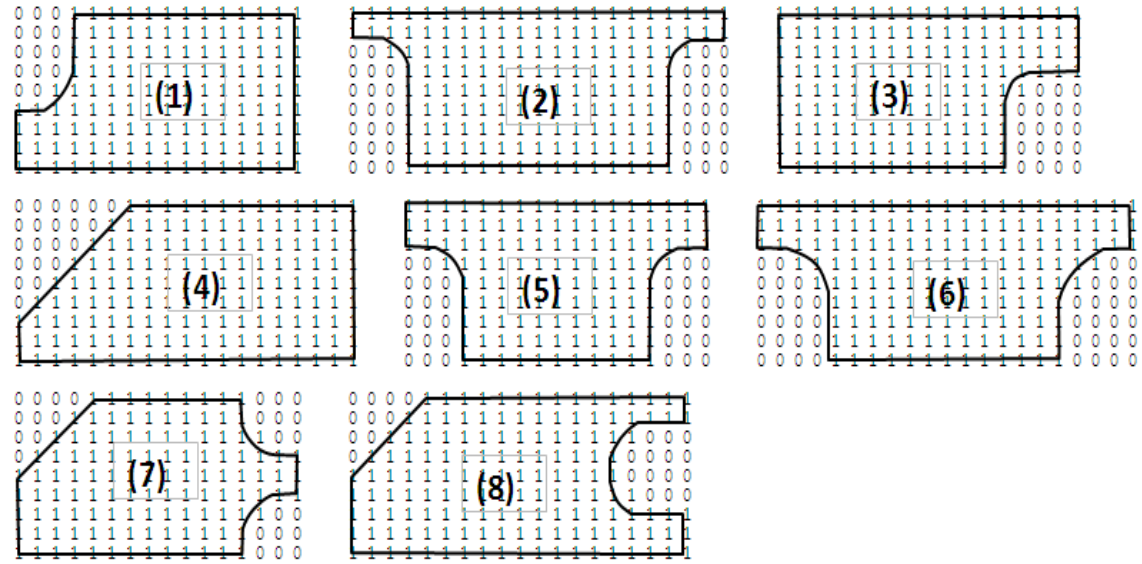

(a)

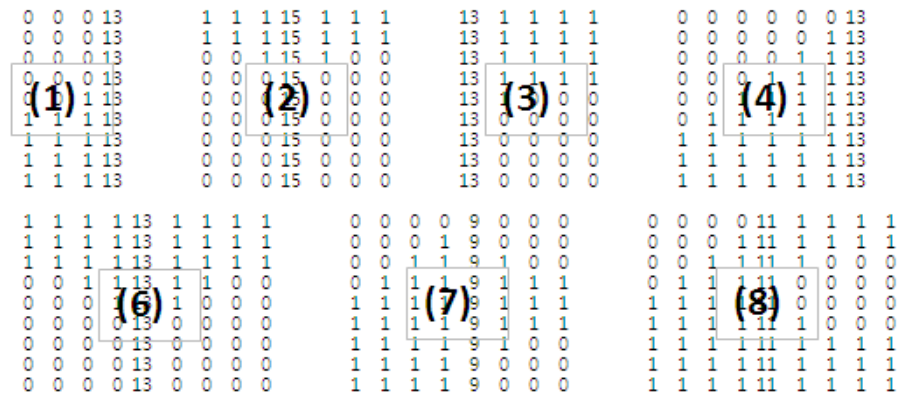

(b)

Figure 7. Shapes for Example 4.2. (a) Actual shapes; (b) Internal representations.

(3) $5_{\text {(6) }}$ (4) (1) (5) $_{\text {(2) }}^{\text {(8) }}$

Figure 8. A solution for Example 4.2, nesting on flat bar. 
this problem can be easily solved by considering the area between the irregular stock and its MER the shapes already assigned positions. Figure 9 shows a solution to an example of nesting on irregular stock. The solution was found at the 93,847 th node.

\subsection{Berth Allocation}

Berth allocation problem is to assign the vessels needing services at specific time at specific locations at quay. Each shape is rectangle ('time $\mathrm{x}$ length') with ES and LF in time axes, between which the shape must be located. The berth allocation is also needed at shipbuilding for out-fit processes at quay area. Scheduling outfitting processes, which require service time of several months, is usually simpler than berth allocation problems at ports. In this example, the lengths of the vessels are given and the process time or service time is fixed. ES and LF are defined for each vessel, which means there is a slack for each service time. In this example, the quay is assumed to have the same water depth and its layout to be straight and continuous.

If the problem does not satisfy the following conditions, no solution exists. The first condition means no single service time crosses the scheduling window, and no vessel is bigger than the length of the quay.

$$
\forall \operatorname{vessel}_{i}\left(\left(\text { length }{ }_{i} \leq \operatorname{Length}_{\text {quay }}\right) \text { and }\left(L S_{i} \geq t_{\text {start }}\right) \text { and }\left(E F_{i} \leq t_{\text {end }}\right)\right)
$$

where, $L S_{i}$ : Latest start time for $\operatorname{vessel}_{i}\left(=L F_{i}-T_{i}\right)$

$E F_{i}$ : Earliest finish time for $\operatorname{vessel}_{i}\left(=E S_{i}+T_{i}\right)$

$T_{i}$ : service time for vessel $i$

$t_{\text {start }}$ and $t_{\text {end }}:$ start time and end time of scheduling window

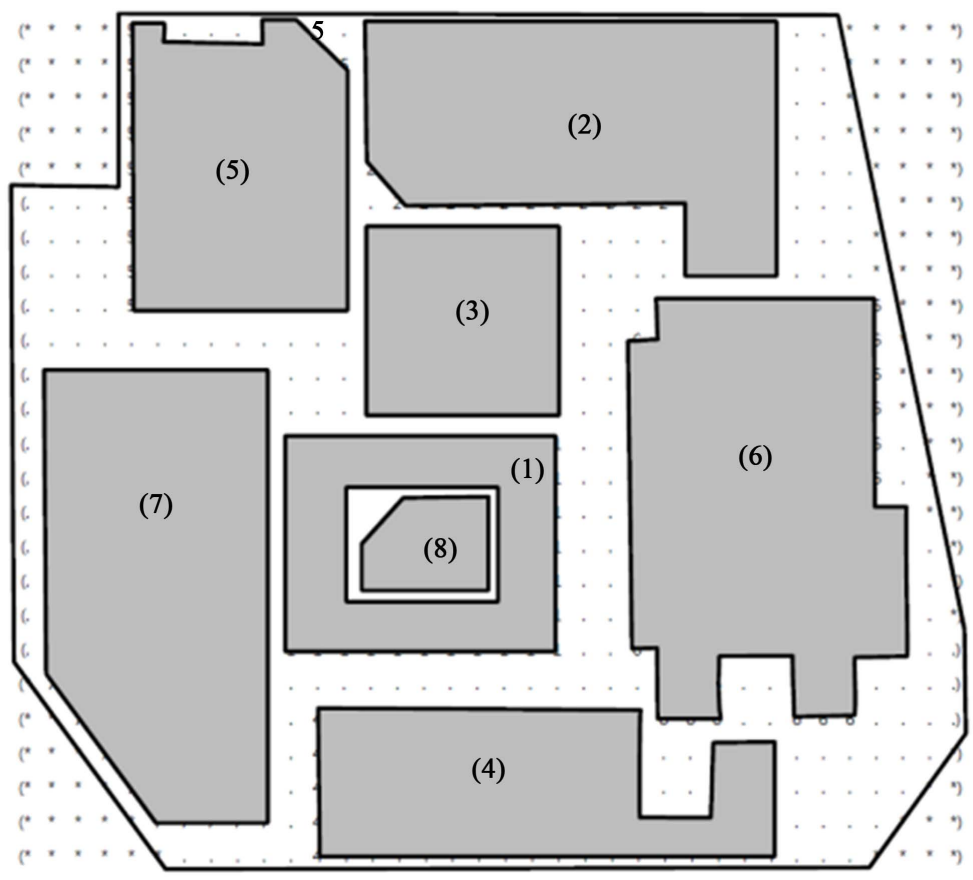

Figure 9. A solution for Example 4.3, nesting on irregular stock. 
The second condition means the sum of the areas of all shapes (time $\mathrm{x}$ length) cannot exceed the size of scheduling window $\mathrm{x}$ the length of quay.

$$
\sum_{i} \text { length }_{i} * T_{i}<\text { Length }_{\text {quay }} *\left(t_{\text {end }}-t_{\text {start }}\right)
$$

The third condition means that at each moment, the sum of the lengths of vessels requiring that moment cannot exceed the length of the quay.

$$
\forall t_{k}\left(\sum_{i \in\{A\}} \text { length }_{i}\right)<\text { Length }_{\text {quay }}
$$

where, $t_{k}$ : time step $k . t_{\text {start }} \leq t_{k} \leq t_{\text {end }}$.

$\{A\}=\left\{j \mid L S_{j} \leq t_{k} \leq E F_{j}\right\}:$ all vessels requiring service at time $t_{k}$

Before start searching, jobs are sorted by ES in ascending order, by length in descending order, and by LF in ascending order. The rationale behind this is "bookshelf rule". In shelving the books, people usually place the tallest and thickest books on one side to make more rooms useful on top of the books. In order to expedite searching, it is reasonable to assign jobs with the earlier ES first. The length and LF are used not only to expedite searching but to maximize the chunk of unassigned area in time-length space in order for the space to be useful.

Figure 10 shows the result of a berth allocation example, in which horizontal axes shows quay area and vertical axes shows time. In Figure 10, each rectangle shows the length of vessel (width) and service time (height). The solid lines show ES and LF of each vessel. The solution was found at the 1782th node.

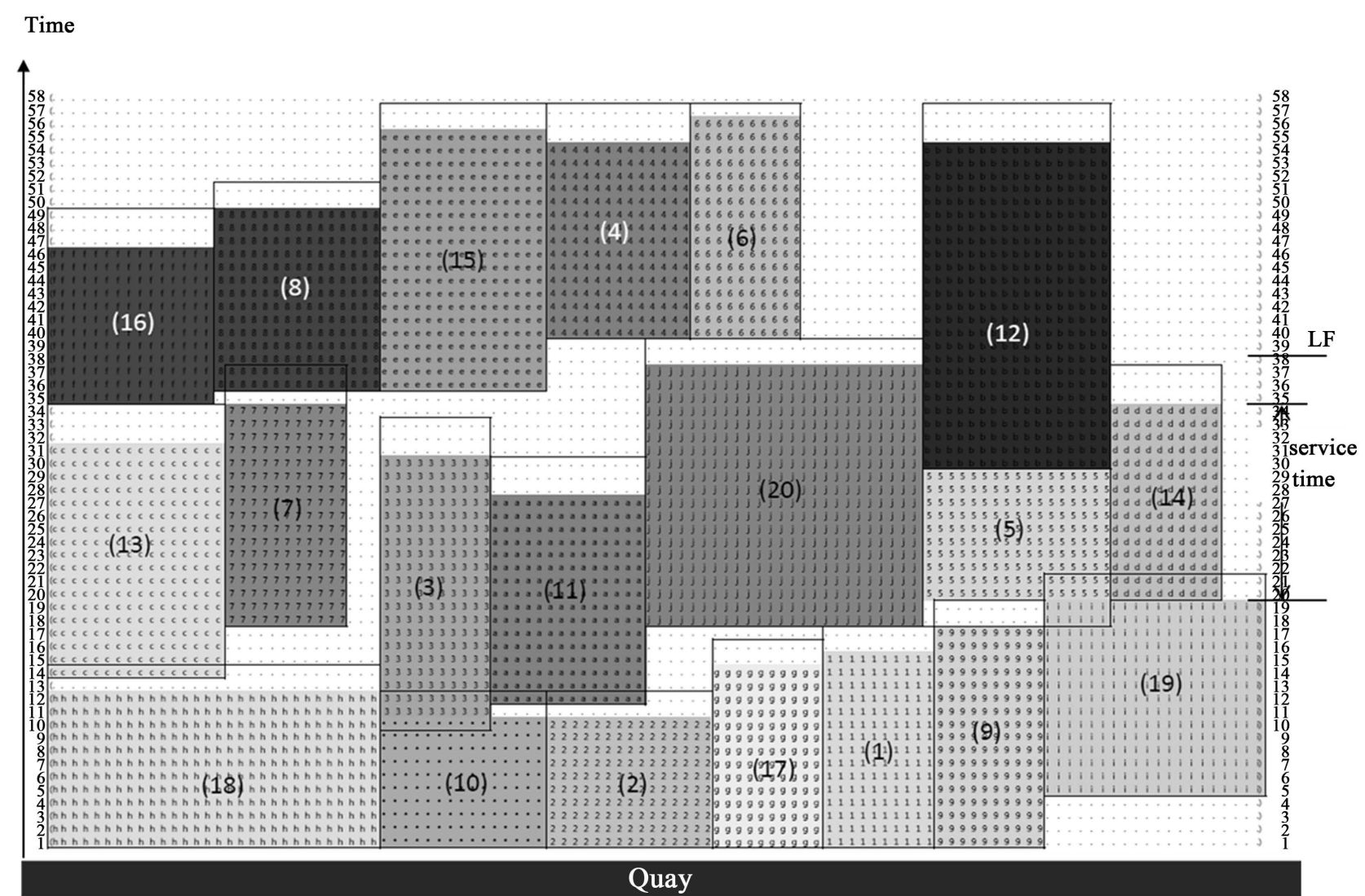

Figure 10. A solution for Example 4.4, berth allocation. 
Berth allocation problem is an NP-hard problem. The search space, however, for scheduling outfitting jobs in shipyard is of reasonable size. In one of the world's biggest shipbuilding companies, about ten vessels are in quay area for outfitting at the same time and the duration of each job is several months. In addition to that, the job has upper and lower limits (LF and ES), which further reduce the search space.

\section{Conclusions}

This paper presents an expert system based on heuristics to solve various two dimensional nesting problems. The system was developed in CLIPS, an expert system shell, and applied to examples for general nesting, nesting on a blank with irregular boundary, nesting on flat-bar in shipbuilding, and birth allocation for outfitting work in shipbuilding. Each application has different constraints so that the system can use different representation of facts and/or utility functions.

The system used pixel representations for shapes with which nesting on a blank with irregular boundary could be easily solved by regarding the area between MER and the boundary of the blank as the shapes already laid. Pixel representation has many advantages over approximate polygon representation in that it can be easily prepared from bit-map images that can be obtained from various sources including CAD systems. The system presented can nest a part inside another hollow part, which is difficult with approximate polygon method. For general nesting, the sum of overlapping areas between a part and the parts already laid on the blank is used as a utility function to maximize the utilization of the blank.

The system can also solve the berth allocation problem, which is to nest the rectangular shapes with "service time $\mathrm{x}$ the length of the vessel" on a blank with planning window in time $x$ the length of quay. A shape has a float equal to LF ES-service time. For berth allocation problems, pre-screening and simple heuristics are used in searching.

Searching becomes slow as the problem space grows. In order to expedite searching, a utility function is used to sort the nodes in expanding the search tree. For larger problem, however, it may be needed to reduce the resolution of pixels to expedite searching. Considering all the constraints and the number of ships to schedule, on the other hand, the search space for scheduling outfitting jobs in shipyard is of reasonable size.

Expert system is a promising approach for nesting and berth allocation problems since they have discrete number of possible solutions and the performance can be improved by easily adding more rules at implementation stage. This research shows that expert system can easily adapt to different kinds of constraints at various application domains.

\section{Acknowledgements}

This work was supported by Defense Acquisition Program Administration and Agency for Defense Development under the contract UD110006MD, Korea. 


\section{References}

[1] Bennell, J.A. and Oliveira, J.F. (2008) The Geometry of Nesting Problems: A Tutorial. European Journal of Operational Research, 184, 397-415.

[2] Burke, E.K., Hellier, R.S.R., Kendall, G. and Whitwell, G. (2007) Complete and Robust No-Fit Polygon Generation for the Irregular Stock Cutting Problem. European Journal of Operational Research, 179, 27-49.

[3] Babu, A.R. and Babu, N.R. (2001) Ageneric Approach for Nesting of 2-D Parts in 2-D Sheets Using Genetic and Heuristic Algorithms. Computer-Aided Design, 33, 879-891.

[4] Weng, W.-C. and Kuo, H.-C. (2011) Irregular stock Cutting System Based on Auto CAD. Advances in Engineering Software, 42, 634-643.

[5] Sherif, S.U., Jawahar, N. and Balamurali, M. (2014) Sequential Optimization Approach for Nesting and Cutting Sequence in Laser Cutting. Journal of Manufacturing Systems, 33, 624-638.

[6] Zheng, J., Jiang, Z., Chen, Q. and Liu, Q. (2011) Spatial Scheduling Algorithm Minimizing Makespan at Block Assembly Shop in Shipbuilding. International Journal of Production Research, 49, 2351-2371. https://doi.org/10.1080/00207541003709536

[7] Koh, S., Logendran, R., Choi, D. and Woo, S. (2011) Spatial Scheduling for ShapeChanging Mega-Blocks in Shipbuilding Company. International Journal of Production Research, 49, 7135-7149. https://doi.org/10.1080/00207543.2010.535863

[8] Bierwirth, C. and Meisel, F. (2010) A Survey of Berth Allocation and Quay Crane Scheduling Problems in Container Terminals. European Journal of Operations Research, 202, 615-627.

[9] Lee, W.-C., Ma, H. and Cheng, B.-W. (2008) A Heuristic for Nesting Problems of Irregular Shapes. Computer-Aided Design, 40, 625-633.

[10] Tay, F.E.H., Chong, T.Y. and Lee, F.C. (2002) Pattern Nesting on Irregular-Shaped Stock Using Genetic Algorithms. Engineering Applications of Artificial Intelligence, 15, 551-558.

[11] Lee, C.S. and Park, G.R. (1996) Automatic Nesting and NC Cutting of Flat-Bar. IE Interfaces, 9, 283-297.

[12] Lee, C.S., Heo, E.-Y., Shim, J.-H., Chen, F.F. and Kim, D.-W. (2013) Ship and Nesting by Pattern Recognition and Group Arrangement. Robotics and ComputerIntegrated Manufacturing, 29, 56-63.

[13] Frojan, P., Correcher, J.F., Alvarez-Valdes, R., Koulouris, G. and Tamarit, J.M. (2015) The Continuous Berth Allocation Problem in a Container Terminal with Multiple Quays. Expert Systems with Applications, 42, 7356-7366. 
Submit or recommend next manuscript to SCIRP and we will provide best service for you:

Accepting pre-submission inquiries through Email, Facebook, LinkedIn, Twitter, etc. A wide selection of journals (inclusive of 9 subjects, more than 200 journals)

Providing 24-hour high-quality service

User-friendly online submission system

Fair and swift peer-review system

Efficient typesetting and proofreading procedure

Display of the result of downloads and visits, as well as the number of cited articles Maximum dissemination of your research work

Submit your manuscript at: http://papersubmission.scirp.org/

Or contact jsea@scirp.org 